\title{
Tratamiento de la Taquicardia Paroxística, del Recién Nacido con Reflejo de la Sumersión
}

\author{
Dres. Alex is Lama T. ${ }^{1}$, Luis Kother R. ${ }^{1}$, Jaine Tapia . $^{2}$ y Humberto Broitman D. ${ }^{3}$
}

\section{Treatment of Supraventricular Tachycardia with the diving reflex in a newborn.}

\begin{abstract}
A twenty five days old newborn with Paroxysmall Supraventricular Tachycardia was reverted to sinus rythm by submerging his face in cold water under continuous electrocadiographic monitoring. This is the first report in Chile of a newborn treated this way.
\end{abstract}

Las intervenciones parasimpáticas, v. gr., la estimulación del seno carotídeo y la compresión de los globos oculares, constituyen un tratamiento ampliamente aceptado de las Taquicardias Paroxísticas Supraventriculares (TPSV) de la infancia ${ }^{1}$

Sin embargo, la estimulación vagal producida al sumergir la cara en agua fría -reflejo de sumersión- aunque reportada como eficaz y segura en el tratamiento de la TPSV del adulto ${ }^{2}, y$ más recientemente en la del niño ${ }^{3}$, ha sido poco difundida.

A continuación, presentamos el primer caso descrito en nuestro medio, de TPSV convertida a

1 Médico Unidad Cuidados Intensivos, Hospital Gmo. Grant Benavente, Concepción.

2 Médico Becado, Servicio de Pediatría Hosp. Gmo. Grant B., Concepción.

3 Médico Pediatra, Unidad Neonatología Servicio Pediatría Hosp. Gmo. Grant B., Concepción. ritmo sinusal con el llamado reflejo de sumersión en un recién nacido.

\section{CASO CLINICO}

Recién nacido de sexo masculino, cesirea a las 35 y media semanas por gestación. Frecuencia Cardiaca fetal sobre $250 \mathrm{x}$ min., una semana antes del parto. Peso de nacimiento 2460 grs.; Apgar 8.9 . 9 - 10. Examen cardíaco normal.

A los 11 días de vida tuvo inquietud y quejido, asociado a tetargo y rechazo de la alimentación. Al dia siguiente ingresó al Hospital Gmo. Grant Benavente de Concepción, estaba hipoactivo pálido, sin cianosis y se alimentaba con dificultad. Al examen físico tenía una frecuencia cardíaca sobre $300 \mathrm{x} \mathrm{min} \mathrm{,,} \mathrm{borde} \mathrm{inferior} \mathrm{del} \mathrm{higado} 5 \mathrm{~cm}$. bajo el borde costal derecho; sin edema ni estertores pulmonares. El electrocardiograma (ECG) demostró una TPSV de $310 \mathrm{x}$ min. que se convirtió a 
ritmo sinusal con una descarga eléctrica de 4 joules.

A los 25 días de vida, reingresó por irritabilidad y palidez. Con latido apical hipequinético, frecuencia cardiaca mayor que $300 \mathrm{x} \mathrm{min.,}$ borde inferior del hígado $4 \mathrm{cms}$. bajo el borde costal derecho, auscultación pulmonar normal, y sin edema. El ECG demostró una TPSV de 300 latidos $\mathrm{x}$ min. con control continuo, en monitor del ECG, se sumergió brevemente la cara del paciente en un recipiente con agua enfriada con hielo hasta aproximadamente 4 grados ${ }^{\circ} \mathrm{C}$. En coincidencia con la maniobra el ritmo se convirtió en sinusal en menos de 2 segundos (Figura 1). No hubo atritmias ni otras complicaciones. El examen card íaco, la radiografía de tórax y el ECG posteriores al procedimiento fueron normales. Se indicó digital en dosis habituales y se dio de alta en buenas condiciones.

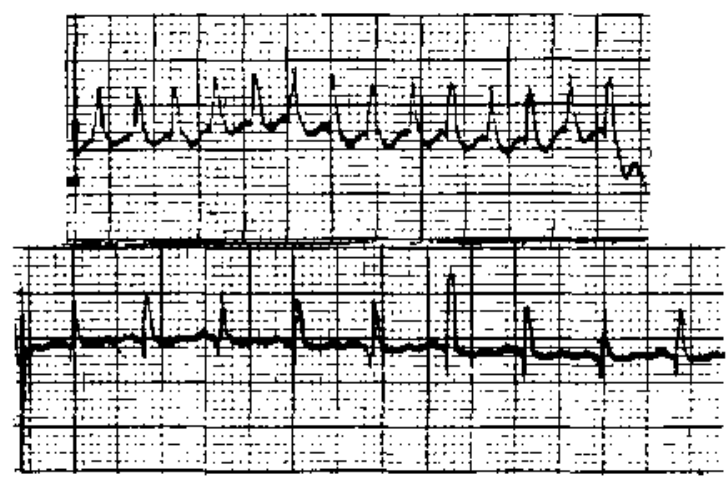

Figuta 1.

EJ trazo superior muestra la taquicardia paroxistica in mediatamente antes de la inmersión. El trazo inferiot, inmediatamente despues, muestra ritmo sinusal.

\section{COMENTARIO}

Las taquiarritmias supraventriculares en el recién nacido no son infrecuentes y pueden, ocasionalmente, significar un problema del manejo ${ }^{4}$.

La insuficiencia cardíaca severa es la forma más frecuente de presentación de la TPSV del recién nacido $^{5}$. La elevada frecuencia cardíaca de la arritmia y el retardo del tratamiento por las dificultades para reconocerla oportunamente por parte de los familiares pueden ser factores responsables.

El manejo clásico de la TPSV de la infancia no difiere fundamentalmente de lo que se hace en el adulto $^{6}$ Se basa en el uso de maniobras vagales (v.gr. estimulación del seno carotídeo y compre- sión ocular), digital y cardioversión eléctrica ${ }^{1}$.

Recientemente, la estimulación vagal provocada por la inmersión de la cabeza en agua con hielo (reflejo de sumersión) ha sido usada con buen éxito para convertir a ritmo sinusal la TPSV de una niña de $8 \operatorname{años}^{3}$, y de dos recién nacidos de 7 y 10 dias de edad?

El reflejo de sumersión, vestigio del existente en el animal acuático, es mediado por la estimulación, con agua fría, de las terminaciones nerviosas ubicadas en el área de la boca y de la nariz, $y$ en parte también por la apnea, produce una compleja respuesta cardiovascular, caracterizada por vasocontricción periférica y disminución de la frecuencia cardíaca con leve aumento de la presión arterial $^{2}$.

Como ocurre con las maniobras vagales convencionales, el reflejo de sumersión puede, ocasionalmente, producir arritmias ventriculares ${ }^{8} ; \sin$ embargo, Wildenthal y Atkins ${ }^{2}$, que han usado este reflejo en más de 60 pacientes adultos, concluyen que el procedimiento es seguro, aparte de convertir más del $90 \%$ de las TPSV. De todas maneras es recomendable efectuar la maniobra bajo control electrocardiográfico permanente.

Debido a la alta tendencia a repetirse que tiene la TPSV del recién nacido ${ }^{\circ}$, estos pacientes, una vez convertidos deben recibir digitallicos, y en aquellos casos en que el reflejo de sumersión produce una rápida conversión y sin complicaciones, se podría instruir a los padres para repetir el procedimiento en sus casas.

El reflejo de sumersión, por ser un método sencillo, indoloro, seguro y rápidamente efectivo ta mayor parte de las veces, debiera incluirse de rutina dentro de las maniobras vagales usadas en el tratamiento de la Taquicardia Paroxística Supraventricular del recién nacido.

\section{RESUMEN}

Se presenta el caso de un recién nacido de 25 dias de edad con una taquicardia paroxistica supraventricular (TPSV), convertida a ritmo sinusal con el llamado "rellejo de sumersión '. Este es el primer recièn nacido con TPSV convertido con este eficaz y sencillo procedimiento que se comunica en nuestro medio.

\section{REFERENCIAS}

${ }^{1}$ Olley P. Corliac Ambythmias, In: Keith J., Row R. and Vad $P$. Heart Discase in infancy and Childhood, Third Edition, Mac Miltan, New York, 1978; Chap. 17 pag. 275. 
2 Wildenthal $K$. and Atkins $J$. Use of the "diving reflex" for the treatment of paroxysmal supraventricular tachycardia. An. Heart J. 98: 536, 1979.

${ }^{3}$ Whitman $V$. and Zakeosian $G$. The diving reflex in termination of supraventricular tachycardia in childhood. J. Pediatr. 89;(6) 1032, 1976.

4 Radford D. Izukawa $T$. and Rowe R.D. Congenital paroxysmal atrial tachycardia. Archives of Disease in Childhood 51:613,1976.

${ }^{5}$ Vera $S_{.}$. Zilleruelo R., Eimbcke F., Vicuña D. y Enriquez G. Arritmias del recién nacido. Rev. Médica Chile 109(12): $\vee ., 1981$ (Resumen).
${ }^{6}$ Lama A., Pacheco V. y Rodriguez M. Taquiarritmias supraventriculares en la infancia. Rev. Médica Chile 109(12): III, 1981 (Resumen).

${ }^{7}$ Whitman V.. Friedmen Z., Berman W. Jr., and Maisels $M . J$. Supraventricular tachycardia in newborn infants: an approach to teraphy. J. Pediatr. 91:304, 1977.

${ }^{8}$ Condry P., Jain A., Morshall R. and Bowyer A. Ventricular tachycardia caused by the diving reflex. Lancet 2 : 1263,1975

9 Lundberg A. Paroxysmal tachycardia in infancy: follow-up study of 47 subjets ranging in age from 10 to 26 years. Pediatrics $51: 26,1973$. 